\title{
An experimental study on the effect of salt spray testing on the optical properties of solar selective absorber coatings produced with different manufacturing technologies
}

\author{
Huai Chen ${ }^{1}$ Wenfeng Gao ${ }^{1}$ (D) Tao Liu ${ }^{1}$ Wenxian $\operatorname{Lin}^{1,2} \cdot$ Ming Li $^{1}$
}

Received: 26 September 2018 / Accepted: 4 March 2019 / Published online: 8 March 2019

(c) The Author(s) 2019

\begin{abstract}
Solar selective absorber coating (SSAC) is one of the key components of a solar collector, with its optical properties having a significant impact on the collector's thermal performance. The key parameters characterizing the optical properties of an SSAC are the solar absorptance (absorptance of solar radiation) and the thermal emittance (emittance for long-wave radiation). However, some of high-performing SSACs suffer from some drawbacks, such as lower durability, lower resistance to corrosion and abrasion, which is particularly harmful for SSACs, as, for example, chlorides in the atmosphere have become a main contributor to corrosion in coastal areas with the increasing trend of global warming. In this paper, salt spray tests have been conducted on the SSACs manufactured by three common manufacturing technologies, i.e., the anode oxidation (AO) technology, the vacuum magnetron sputtering (VMS) technology, and the black chromium plating (BCP) technology, over the testing durations of $12 \mathrm{~h}, 24 \mathrm{~h}, 36 \mathrm{~h}$, and $48 \mathrm{~h}$, respectively, to examine the effect of the salt spray testing on the optical properties of SSACs manufactured by different manufacturing technologies. The salt spray testing is an accelerated aging testing method for evaluating the SSAC's resistance to corrosion when it is under an extended exposure to a saline, or salted, spray (fog). The experimental results show that, in general, the SSACs manufactured by the BCP technology have excellent resistance to salt spray (i.e., to corrosion) and those manufactured by the AO technology have only reasonable resistance to corrosion, whereas the SSACs manufactured by the VMS technology have very poor resistance to corrosion. The results also demonstrate that there are noticeable differences in the optical properties of the SSAC samples even manufactured by the same technology but by different manufacturers, with some having significant differences. The causes for the differences have been further examined through the inspection of the physical appearance of the selected SSAC samples and the experimentally measured distributions of the monochromatic reflectance of solar radiation of the samples over the solar spectrum before and after the salt spray testing over different durations.
\end{abstract}

Keywords Salt spray testing $\cdot$ Solar selective absorber coating $\cdot$ Corrosion $\cdot$ Resistance $\cdot$ Solar absorptance $\cdot$ Thermal emittance

\section{Introduction}

The solar selective absorber coating (hereinafter abbreviated as "SSAC") on the absorber plate of a flat-plate solar collector plays a crucial role in dictating the thermal performance

Wenfeng Gao

wenfenggao@163.com

1 Solar Energy Research Institute, Yunnan Normal University, Kunming 650092, Yunnan, China

2 College of Science and Engineering, James Cook University, Townsville QLD 4811, Australia of the collector. An SSAC is considered efficient if it has a very high solar absorptance over the solar spectrum (200-2000 nm) to maximize the absorption of solar radiation and a very low thermal emittance to minimize the longwave thermal radiative heat loss. Compared with a traditional coating which can have a very high solar radiation absorptance but a very high thermal radiation emittance, an SSAC has better photo-thermal conversion efficiency. Hence, the solar absorptance, $\alpha$, and the thermal emittance, $\varepsilon$, are the two key parameters characterizing the optical properties of an SSAC and its associated photo-thermal conversion performance $[1,2]$. 
At present, SSACs are produced mainly by three common manufacturing technologies; the anode oxidation technology (hereinafter abbreviated as "AO technology"), the vacuum magnetron sputtering technology (hereinafter abbreviated as "VMS technology"), and the black chromium plating technology (hereinafter abbreviated as "BCP technology"). The AO technology is the most popular for flat-plate solar collectors due to relatively low coasts, good optical properties (with $\alpha$ as high as more than 0.92 and $\varepsilon$ less than 0.2), and sound climate resistance of the SSACs manufactured. The SSACs produced by the BCP technology possess superior optical properties (with $\alpha$ as high as 0.95 and $\varepsilon$ less than 0.1 ) and excellent resistance to heat, humidity, and climate. However, one drawback of the BCP technology is the potential pollution to the environment of the waste liquid produced in the manufacturing processes, if it is not properly controlled. Nevertheless, such potential pollution may be avoided by controlling and improving the manufacturing processes. The VMP technology is the latest and new-generation technology to manufacture SSACs with excellent optical properties (with $\alpha$ as high as 0.95 and $\varepsilon$ less than 0.06 ) and good resistance to heat and humidity. In addition, the risks of polluting the environment are significantly reduced when compared to the BCP technology.

The life span of a flat-plate solar collector is about 20-30 years. Furthermore, as the SSAC is exposed to air, corrosion will occur on the SSAC, which will inevitably have adverse effects on the solar absorptance and the thermal emittance of the SSAC, leading to dramatic degradation of the thermal performance of the solar collector. Solar thermal collectors have to withstand conditions like high temperatures, high humidity, ultraviolet irradiance, or wind and snow loads depending on the geographic position and atmospheric corrosivity [3-9]. As the key component of a solar thermal collector, the optical properties of SSACs are negatively affected by such conditions [3]. Hence, the main challenges faced by research and development of SSACs are to improve the optical properties, but at the same time to enhance reliability and durability [10-12]. The durability of SSACs has been a main concern in the last two decades and accelerated aging tests were developed to ensure a life span of at least 25 years $[4,10,11,13-16]$. Presently, these tests are incorporated in the International Standard ISO 22975$3: 2014[12,17]$. The experimental design of the tests was made based on some thorough studies on the degradation of SSACs $[10,12,18,19]$. In this standard, only high temperature, condensed water, and high humidity air containing sulfur dioxide are considered in the tests for determining the reliability and durability of a SSAC [17]. However, numerous countries have extensive coastal areas, hence the reliability and particularly high durability of SSACs to salt, which causes high corrosion due to high chlorides levels at the areas, is also a primary factor that should be considered as well [3, 20, 21].

The SSAC's resistance to corrosion in coastal areas can then be evaluated by salt spray testing, which is an accelerated aging test used to evaluate the coating's resistance to corrosion under an extended exposure to a saline, or salted, spray (fog) [22]. Salt spray testing is conducted in a closed salt spray chamber where the sample is subjected to a sodium chloride fog, an extremely corrosive atmosphere. The length of exposure time is dependent on the material, the coating, and the standard. After exposure, the sample is examined to evaluate its corrosion-resistance performance.

Salt spray testing on SSACs has been reported by some studies. Fan et al. conducted a salt spray testing on black chromium coatings, anode oxidation coatings, and blue titanium coatings, and concluded that the black chromium coatings have the best resistance to salt spray, the blue titanium coatings have the worst resistance, and the coatings produced by the physical vapor deposition technology have very poor resistance to salt spray [23]. Yao et al. studied the resistance to salt spray of several SSACs produced by different manufacturing technologies and examined the relations between the SSACs' resistance to salt spray and the compositions of the coatings and the manufacturing technologies [24]. Yin et al. used the Chinese National Standard GB/T 1771-2007 to explore the causes for the stripping of SSACs through an immersion test of the SSACs in seawater [25, 26].

An examination of the current literature has revealed that previous studies on the SSAC's resistance to salt spray have mainly focused on the comparison of the SSAC's solar absorptance and thermal emittance before and after the salt spray testing over $48 \mathrm{~h}$. To examine the effects of the duration for salt spray testing on the optical properties of SSACs produced by different manufacturing technologies, experiments have been carried out in this paper to study the resistance to salt spray of the SSACs produced by the three common manufacturing technologies, i.e., the AO technology, the VMS technology, and the BCP technology, over the testing durations of $12 \mathrm{~h}, 24 \mathrm{~h}, 36 \mathrm{~h}$, and $48 \mathrm{~h}$, respectively.

\section{Experimental methods and apparatus}

\section{Experimental methods and apparatus for $a$ and $\varepsilon$}

As mentioned above, the key parameters characterizing the optical properties of an SSAC are the solar absorptance, $\alpha$, and the thermal emittance, $\varepsilon$. A good SSAC is the one that has a value of $\alpha$ over the solar spectrum (250-2500 nm) which is as high as possible, but at the same time has a value of $\varepsilon$ over the mid- and far-infrared spectrum $(2-100 \mu \mathrm{m})$ which is as low as possible. 
The solar absorptance of an SSAC, $\alpha$, is determined experimentally by $\alpha=1-\rho$, where $\rho$ is the reflection of solar radiation of the SSAC. The wavelengths of solar radiation are mainly over the range of 250-2500 nm (i.e., the solar spectrum). If this wavelength range is equally divided into 50 sections, $\alpha$ can be estimated by [27]

$\alpha=1-\sum_{i=1}^{50} \rho_{\lambda_{i}} \cdot 2 \%$

where $\rho_{\lambda_{i}}$ is the average monochromatic reflectance of solar radiation of the SSAC over the $i$ th wavelength range section. In this paper, $\alpha$ is calculated using Eq. (1) by experimentally measuring $\rho_{\lambda_{i}}$ for each SSAC tested using a Cary5000 spectrophotometer, which is shown in Fig. 1, in accordance with the International Standard ISO 22975-3:2014 [17].

The thermal emittance of a surface, $\varepsilon$, is determined by $[27,28]$

$\varepsilon=\varepsilon_{\mathrm{bn}} \frac{\phi_{\mathrm{s}}}{\phi_{\mathrm{b}}}$

where $\phi_{\mathrm{s}}$ is the measured thermal radiation intensity of the surface sample at a preset temperature, $\phi_{\mathrm{b}}$ is the measured thermal radiation intensity of the blackbody at the same preset temperature, and $\varepsilon_{\mathrm{bn}}$ is the thermal emittance of the blackbody in the normal direction, respectively.

As $\varepsilon_{\text {bn }}$ is 1 , Eq. (2) can then be written as

$\varepsilon=\frac{\phi_{\mathrm{s}}}{\phi_{\mathrm{b}}}$

In an actual experiment to measure $\varepsilon$, a zero calibration cavity is used which has the measured thermal radiation intensity, $\phi_{0}$, and hence both $\phi_{\mathrm{s}}$ and $\phi_{\mathrm{b}}$ must be calibrated with $\phi_{0}$, i.e., $\varepsilon$ is determined experimentally by

$\varepsilon=\frac{\phi_{\mathrm{s}}-\phi_{0}}{\phi_{\mathrm{b}}-\phi_{0}}$.

The apparatus for measuring $\varepsilon$ in this paper was manufactured by Beijing Tianyude Science and Technology Ltd Co. The photos of this experimental apparatus and some of its key elements are presented in Fig. 2. The apparatus is composed of a blackbody cavity, a sample cavity, a zero calibration cavity, a thermic element cavity, and a thermostat water bath, as schematically shown in Fig. 3. The working fluids for the blackbody cavity and the sample cavity are hot water, whereas the working fluids for the zero calibration cavity and the thermic element cavity are cold water.

Before the experimental test to measure $\varepsilon$, the sample is placed in the sample chamber, followed by opening the thermostat water bath to pump hot water to circulate through the sample cavity and the blackbody cavity. The zero calibration cavity and the thermic element cavity are filled with cold water. When the temperature of the sample attains the preset value, the experimental test commences. The first step of the experiment is to place the zero calibration cavity on the thermic element and to read the thermal radiation intensity for the zero calibration cavity, $\phi_{0}$, from the computer that connects with the secondary instrument which converts the acquired signals into readable data. The second step is to replace the zero calibration cavity by the sample cavity and to read the thermal radiation intensity of the surface of sample, $\phi_{\mathrm{s}}$. The final step is to replace the sample cavity by the blackbody cavity and to read the thermal radiation intensity of the blackbody cavity, $\phi_{\mathrm{b}}$.
Fig. 1 A photo of the Cary5000 spectrophotometer

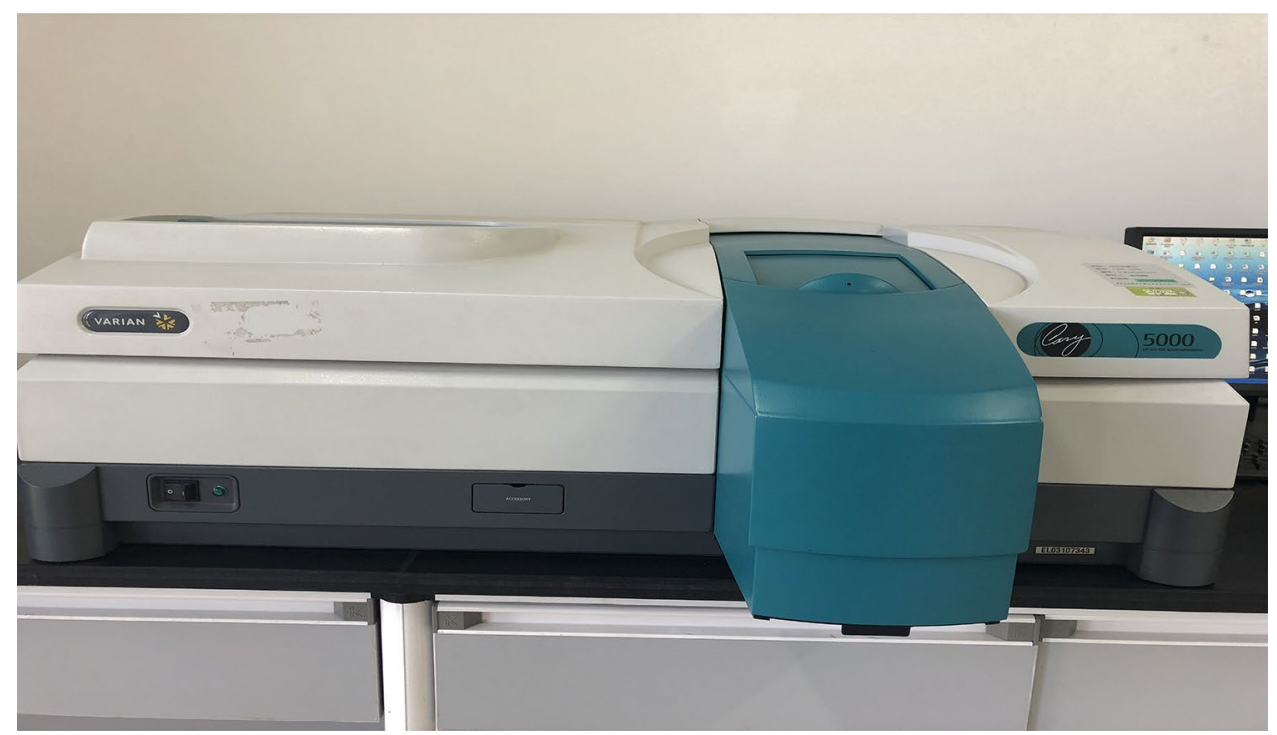




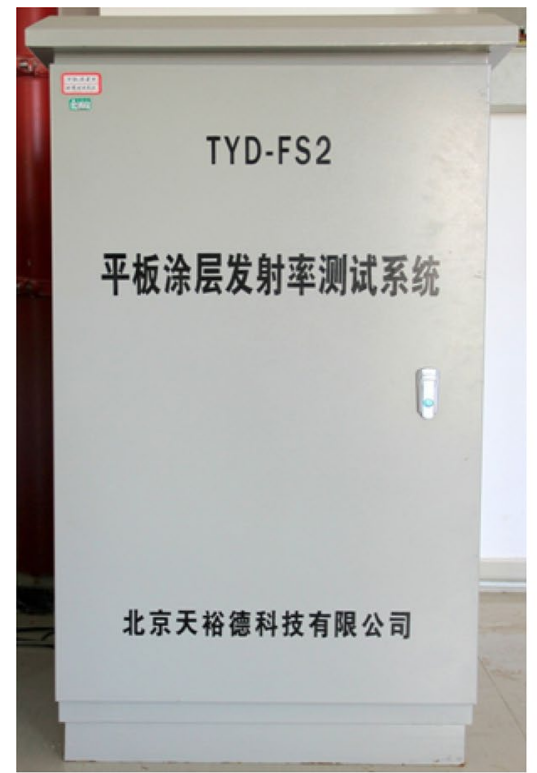

(a)

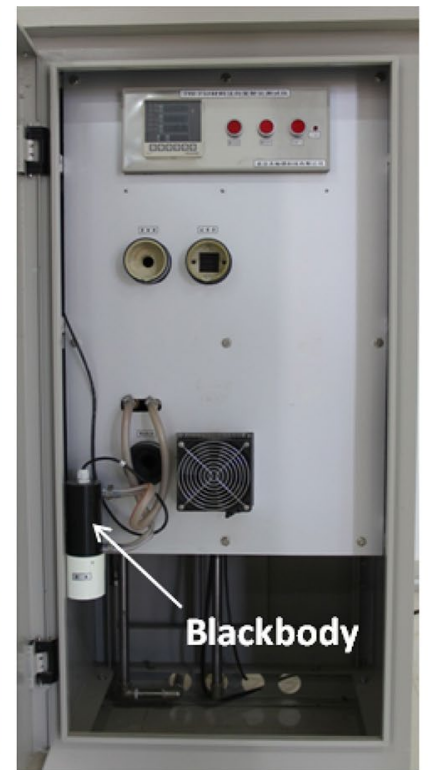

(b)

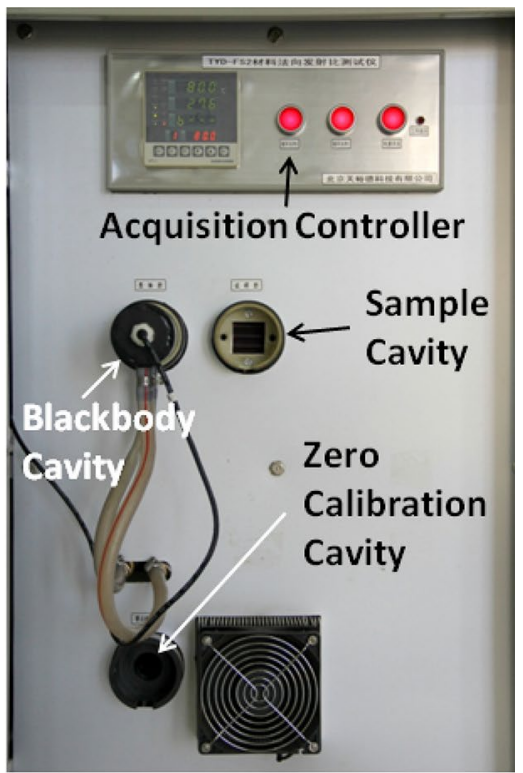

(c)
Fig. 2 The photos of the experimental apparatus for measuring $\varepsilon$ which was manufactured by Beijing Tianyude Science and Technology Ltd Co.: a the frontal cover and the outside of the apparatus, b the inside of the apparatus and the blackbody, $\mathbf{c}$ the locations of the sample cavity, the blackbody cavity, the zero calibration cavity, and the acquisition controller inside the apparatus
Fig. 3 A schematic of the experimental apparatus for measuring $\varepsilon$ : 1 - the blackbody cavity, 2-the zero calibration cavity, 3-the sample cavity, 4-the sample, 5-the thermic element, 6 - the thermic element cavity, 7-the thermostat water bath, and 8 - the secondary instrument which is connected to a computer (not shown) to acquire and record the measured data
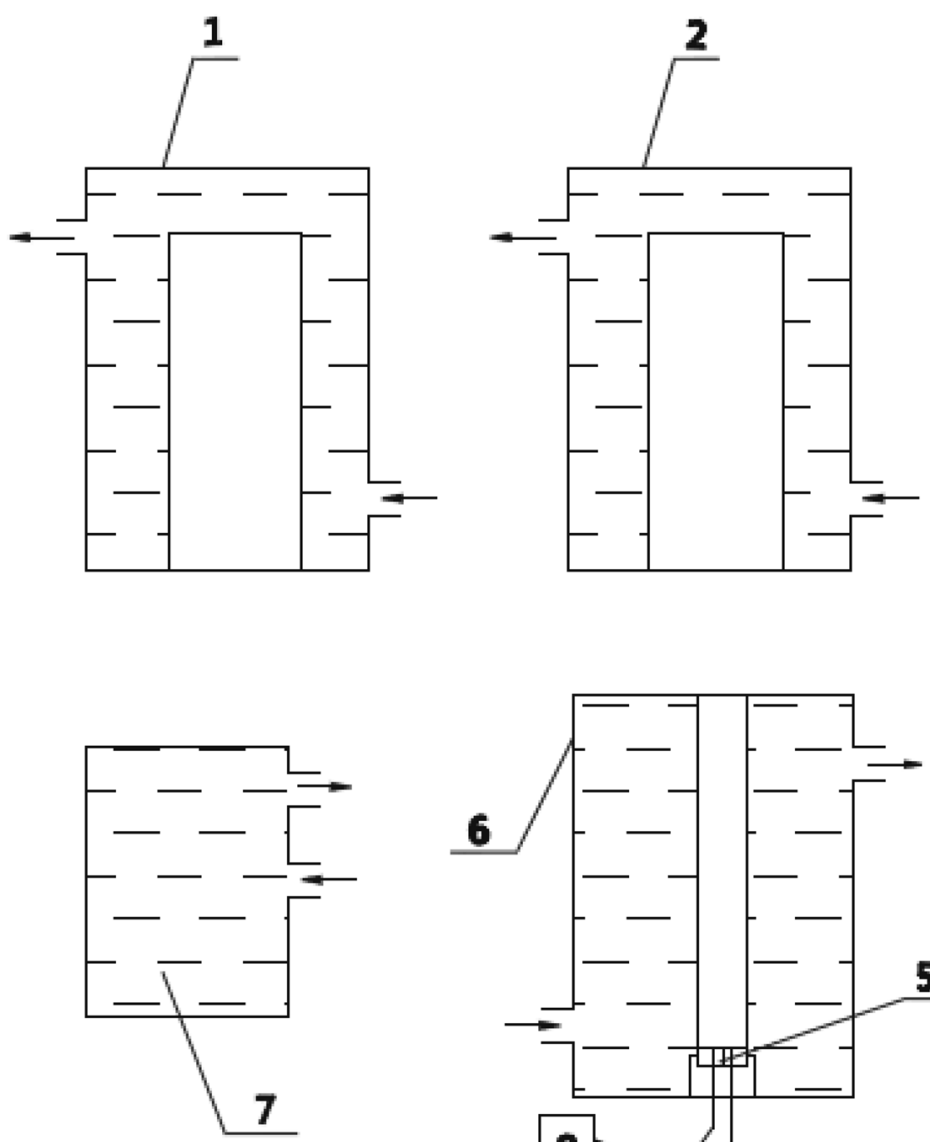
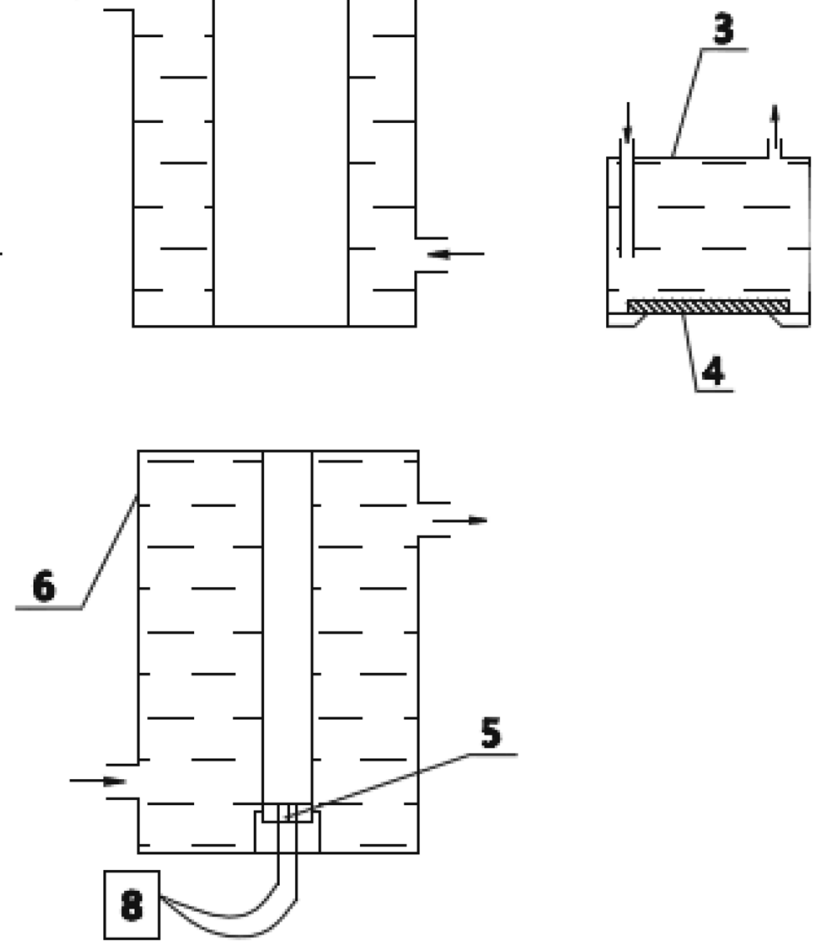


\section{Experimental methods and apparatus for salt spray testing}

Based on the International Standard ISO 22975-3:2014 and the Chinese National Standard GB/T 1771-2007, the experimental scheme consists of the following key steps $[17,26]$ : firstly, a smooth and clean SSAC sample without any scratch is taken from the absorber of a flat-plate solar collector supplied by the manufacturer and is prepared with the required size suitable for testing; the testing solution is then prepared and poured into the saline tank as soon as possible to avoid a prolonged stay outside the tank which may affect the solution concentration and in turn the experimental results; subsequently, the prepared SSAC sample is placed in the salt spray testing chamber at a specific angle and the salt spray testing device is switched on to start the test; after the testing, the SSAC sample is cleaned with water and then dried up; and finally the optical properties of the SSAC sample are measured and analyzed to determine the values of its $\alpha$ and $\varepsilon$ with the measuring methods and apparatus outlined above.

The testing solution was prepared by dissolving the analytical reagent sodium chloride with the mass fraction no less than $99.5 \%$ into the Grade 3 purified water [29]. The resultant testing solution had a mass concentration of $(50 \pm 5) \mathrm{g} / \mathrm{L}$ and the $\mathrm{pH}$ value of $6.5-7.2$. The flow rate of the salt spray fog produced was $1-2.5 \mathrm{~mL} / \mathrm{h}$.

The salt spray testing was conducted under the following specific testing conditions:
- The temperature in the salt spray testing chamber: $35 \pm 2{ }^{\circ} \mathrm{C}$.

- The working pressure: $1 \mathrm{~kg} / \mathrm{m}^{2}$.

- The placement of the SSAC sample: the surface of the SSAC sample was placed upward in the salt spray testing chamber at an angle of $20 \pm 5^{\circ}$ to the vertical direction.

The experimental setup is shown in Fig. 4, which consists of a salt spray chamber and an air compressor. The salt spray testing was conducted in the salt spray chamber (model F-120A) developed by Dongguan JingZhuo Equipment Co. Ltd. The chamber consists of an inner chamber of the dimensions of $1050 \mathrm{~mm} \times 800 \mathrm{~mm} \times 550 \mathrm{~mm}$ (length $\times$ width $\times$ height), with the working temperature over the range $0-99.9 \pm 0.3{ }^{\circ} \mathrm{C}$ and the flow rate of salt spray fog of $1-2.5 \mathrm{~mL} / \mathrm{h}$, the thermostatic controllers controlling the temperature in the chamber, the spray device which can provide salt spray by the combination of an air compressor, a solution storage tank, and several nozzles, as well as testing plate brackets.

When the experiment commences, the air compressor starts to compress air to activate the spray device. Meanwhile, the working pressure and temperature can be set on the control panel. By controlling the working pressure, the flow rate of salt spray can be produced in the required range. The samples are then placed into the inner chamber on the testing plate brackets when the experimental parameters meet the requirements. To maintain the working pressure and temperature, the cover of chamber must be closed quickly after finishing the placement of samples.
Fig. 4 A photo of the experimental setup used for the salt spray testing, which includes a salt spray chamber (model F-120A) and an air compressor

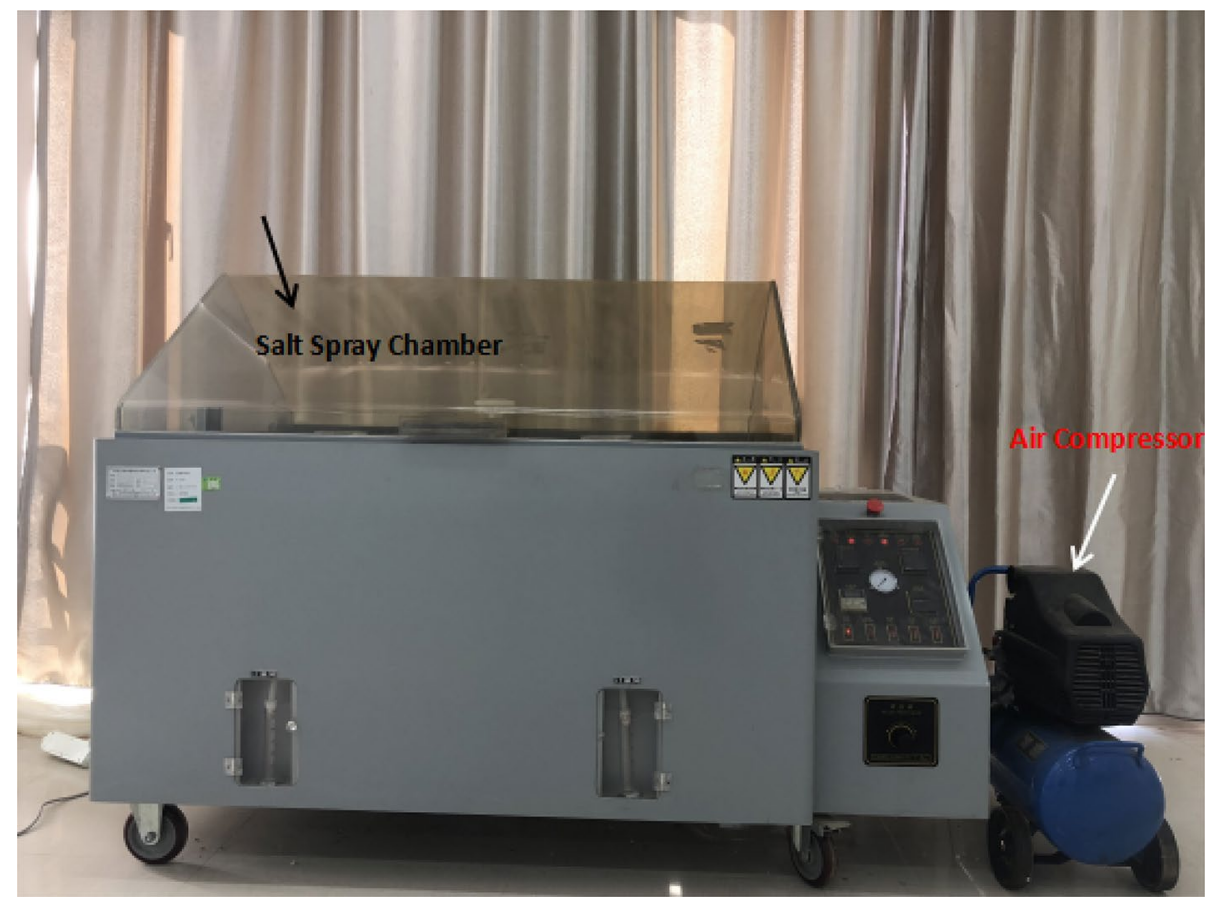




\section{Experimental results and discussions}

In this paper, the optical properties of the SSACs manufactured by the AO technology, the VMS technology, and the $\mathrm{BCP}$ technology are examined by salt spray testing over the testing durations of $12 \mathrm{~h}, 24 \mathrm{~h}, 36 \mathrm{~h}$, and $48 \mathrm{~h}$, respectively, using the experimental methods and apparatus detailed in the previous section, to evaluate the effect of the salt spray testing on the optical properties of SSACs. With such salt spray testing, the SSACs' resistance to corrosion under an extended exposure to an adverse environment, by way of a saline, or salted, spray (fog), can be evaluated by the accelerated aging method.

The six SSAC samples, each with the same size of $40 \mathrm{~mm} \times 40 \mathrm{~mm}$ and labeled as Sample A to Sample F, respectively, were prepared from the absorbers of the flatplate solar collectors supplied by six different manufacturers. The substrates of all these coatings are aluminum. Samples A and B were manufactured with the AO technology, Samples $\mathrm{C}$ and $\mathrm{D}$ were manufactured with the VMS technology, and Samples E and F were manufactured with the BCP technology, respectively. For each sample, a salt spray test was carried out over the testing durations of $12 \mathrm{~h}, 24 \mathrm{~h}, 36 \mathrm{~h}$, and $48 \mathrm{~h}$, respectively. During the tests, the ambient temperature was $23 \pm 2{ }^{\circ} \mathrm{C}$ and the relative humidity was $50 \pm 5 \%$. All the tests were conducted with good air ventilation and without direct exposure to sunlight.

\section{Values of $a$ and $\varepsilon$ of the six SSAC samples}

The experimentally obtained values of $\alpha$ and $\varepsilon$ of the six SSAC samples before and after the salt spray testing over different testing durations are summarized in Table 1. From the table it is seen that before the testing, the values of $\alpha$ for all six SSAC samples were no less than 0.92 and the values of $\varepsilon$ were not greater than 0.2 , indicating that all of them had good optical properties. It is further observed that, among the six SSACs, those with the BCP technology had the best overall optical properties before the testing, with the highest $\alpha$ (more than 0.96 ) and a moderate $\varepsilon$, the SSACs with the VMS technology had the lowest thermal emittance before the testing, although their solar absorptance was the lowest as well, and the SSACs with the AO technology had the comparable optical properties to those with the VMS technology before the testing, in particular Sample B.

The errors caused by the uncertainties associated with the spectrophotometer measuring $\alpha$ and the apparatus for measuring $\varepsilon$ are smaller than 0.0015 and 0.002 , respectively. The accuracy clearly meets the performance test requirements.

The high $\alpha$ values of the six SSAC samples and their differences before the testing can be further examined by their individual distributions of the monochromatic reflectance of solar radiation over the solar spectrum $(250-2500 \mathrm{~nm})$, as shown in Fig. 5, which were measured experimentally before the salt spray testing for each SSAC sample. It is seen that in general, all six SSAC samples had very small values of $\rho_{\lambda_{i}}$ $(\leq 5 \%)$ over the visible light region $(390-780 \mathrm{~nm})$ where the bulk of the solar radiation resides, which explains why all six samples had high $\alpha$ values. The causes for the differences in $\alpha$ of the six SSAC samples, although not large (within 5.5\%), are clearly demonstrated by the significantly different distributions of $\rho_{\lambda_{i}}$ outside the visible light region, in particular beyond $900 \mathrm{~nm}$. Sample E and Sample F had very low $\rho_{\lambda_{i}}$

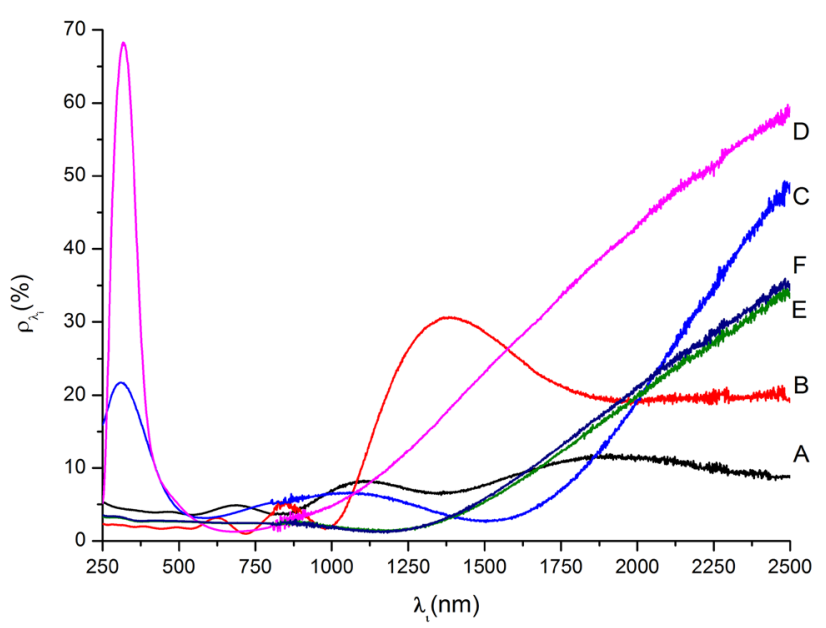

Fig. 5 The experimentally measured distributions of the monochromatic reflectance of solar radiation of the six SSAC samples over the solar spectrum before the salt spray testing
Table 1 Experimentally obtained values of $\alpha$ and $\varepsilon$ for the six SSAC samples before $(0 \mathrm{~h})$ and after the salt spray testing over the testing durations of $12 \mathrm{~h}, 24 \mathrm{~h}, 36 \mathrm{~h}$, and $48 \mathrm{~h}$, respectively

\begin{tabular}{|c|c|c|c|c|c|c|c|c|c|c|c|}
\hline \multirow[t]{2}{*}{ Samples } & \multicolumn{2}{|l|}{$0 \mathrm{~h}$} & \multicolumn{2}{|l|}{$12 \mathrm{~h}$} & \multicolumn{2}{|l|}{$24 \mathrm{~h}$} & \multicolumn{2}{|l|}{$36 \mathrm{~h}$} & \multicolumn{2}{|l|}{$48 \mathrm{~h}$} & \multirow[t]{2}{*}{ Technology } \\
\hline & $\alpha$ & $\varepsilon$ & $\alpha$ & $\varepsilon$ & $\alpha$ & $\varepsilon$ & $\alpha$ & $\varepsilon$ & $\alpha$ & $\varepsilon$ & \\
\hline A & 0.948 & 0.200 & 0.947 & 0.225 & 0.947 & 0.228 & 0.947 & 0.228 & 0.947 & 0.234 & $\mathrm{AO}$ \\
\hline B & 0.930 & 0.113 & 0.922 & 0.180 & 0.922 & 0.180 & 0.922 & 0.180 & 0.922 & 0.180 & $\mathrm{AO}$ \\
\hline $\mathrm{C}$ & 0.933 & 0.100 & 0.875 & 0.320 & 0.862 & 0.392 & 0.756 & 0.536 & 0.663 & 0.679 & VMS \\
\hline D & 0.921 & 0.100 & 0.904 & 0.114 & 0.904 & 0.161 & 0.901 & 0.161 & 0.868 & 0.218 & VMS \\
\hline E & 0.963 & 0.121 & 0.963 & 0.129 & 0.963 & 0.131 & 0.962 & 0.131 & 0.962 & 0.131 & BCP \\
\hline $\mathrm{F}$ & 0.971 & 0.121 & 0.970 & 0.121 & 0.970 & 0.130 & 0.970 & 0.130 & 0.970 & 0.130 & BCP \\
\hline
\end{tabular}


values from 300 to $1300 \mathrm{~nm}$ and their $\rho_{\lambda_{i}}$ values began to increase, monotonically and linearly with large rates, only from about $1300 \mathrm{~nm}$. As the contribution to $\alpha$ from this near-infrared region is negligible, and their differences in $\rho_{\lambda_{i}}$ over the whole solar spectrum are very small, both Sample E and Sample F had very high $\alpha$ values, at 0.963 and 0.971 , respectively. Sample A and Sample B had quite different distributions of $\rho_{\lambda_{i}}$ beyond the visible light region, with Sample B having much larger $\rho_{\lambda_{i}}$ values than Sample A, which is the reason why Sample B's $\alpha$ value was about $2 \%$ lower than that for Sample A. Furthermore, the $\rho_{\lambda_{i}}$ values of both Sample A and Sample B began to increase at smaller wavelengths (at about $900 \mathrm{~nm}$ and $1000 \mathrm{~nm}$, respectively) than those for Samples E and F, which, together with their slight differences over the visible light region, led to smaller $\alpha$ values in Samples A and B when compared to Samples $\mathrm{E}$ and $\mathrm{F}$. The distributions of $\rho_{\lambda_{i}}$ for Samples $\mathrm{C}$ and D are enormously different from those for the other samples. Both samples had large $\rho_{\lambda_{i}}$ values at the lower end of the solar spectrum, in particular for Sample D. They also had large $\rho_{\lambda_{i}}$ values beyond the visible light region, which increased significantly with the wavelength. All these contribute to their relatively smaller $\alpha$ values than the other samples. In particular, the very large $\rho_{\lambda_{i}}$ values at both the lower end of the solar spectrum and beyond the visible light region make Sample D have the smallest $\alpha$ value, at 0.921 , among the six samples.

After the salt spray testing, the values of $\alpha$ for Samples $\mathrm{A}, \mathrm{E}$, and $\mathrm{F}$ were essentially the same as their respective values before the testing, and Sample B also had only a very slight reduction (from 0.930 to 0.922 ), indicating that the salt spray had little effect on the solar absorptance of the SSACs with the AO and BCP technologies. However, the values of $\alpha$ for Sample C reduced significantly after the salt spray testing and the reduction rate in $\alpha$ increased generally when the testing duration was prolonged, at $-5.4 \%,-2.3 \%$, $-11.6 \%$, and $-13.2 \%$ after the durations of $12-\mathrm{h}, 24-\mathrm{h}$, 36-h, and 48-h testing, respectively. A noticeable reduction in $\alpha$ is also found for Sample D, with a 5.5\% reduction in $\alpha$ after the 48-h testing. These results show that in general, the salt spray has a significant impact on the solar absorptance of the SSACs with the VMS technology.

The values of $\varepsilon$ for Samples E and F had small changes before and after the salt spray testing, as the value of $\varepsilon$ for Sample E only had the maximum increase of $8.3 \%$ after the 48-h testing, whereas the increase of $\varepsilon$ for Sample $\mathrm{F}$ was even smaller, with the maximum increase of $7.4 \%$ after the 48-h testing, which indicates that the salt spray only has a minor effect on the thermal emittance of the SSACs with the BCP technology. Although the values of $\varepsilon$ for Samples $A$ and $B$ changed very slightly across different durations of testing, they were considerably larger than their respective values before the testing, in particular for Sample B as its $\varepsilon$ value jumped by $63.6 \%$, implying that the salt spray has a significant effect on the thermal emittance of the SSACs with the $\mathrm{AO}$ technology. Very significant increases in $\varepsilon$ were observed for Samples C and D and their $\varepsilon$ values increased enormously when the duration of testing was prolonged, in particular for Sample $C$ which had its $\varepsilon$ value increased almost sixfold, from 0.100 before the testing to 0.679 after the 48-h duration of testing. This clearly demonstrates that the salt spray has an immerse effect on the thermal emittance of the SSACs with the VMS technology.

Hence, in terms of the overall optical properties, when both the values of $\alpha$ and $\varepsilon$ are considered, it can be concluded that in general the SSACs with the BCP technology have excellent resistance to salt spray (thus to corrosion), and those with the AO technology have only reasonable resistance to salt spray corrosion whereas the SSACs with the VMS technology have very poor resistance to salt spray corrosion.

The results from the table also show that there are differences in measured values of $\alpha$ and $\varepsilon$ for the SSAC samples with the same technology but manufactured by different manufacturers, and some of these differences are significant. Although both Sample A and Sample B were manufactured with the AO technology, the former was manufactured by the traditional AO coating on individual strips in the open air, with the resultant coated strips which can then be directly welded to the risers to form a flat-plate collector, whereas the latter was manufactured by the continuous AO coating on a wide metal roll in a closed chamber, with the resultant coated roll to be cut into desired coated sheets to form a flat-plate collector. Both samples had comparable values of $\alpha$ before the testing and these values changed only very slightly after the testing. However, Sample B had the value of $\varepsilon$ which is much smaller than that for Sample A before the testing; but after the testing, the performance of Sample $\mathrm{B}$, in terms of $\varepsilon$, deteriorated more significantly than that of Sample A. Both Sample C and Sample D were manufactured by the continuous VMS coating on a wide metal roll in a closed chamber, but their resistances to salt spray, in terms of both $\alpha$ and $\varepsilon$, vary enormously. Both samples had the same low thermal emittance and almost the same solar absorptance before the testing; however, the deterioration in the optical properties of Sample C after the testing was more profound than that for Sample $\mathrm{D}$, as the reduction in $\alpha$ and the increase in $\varepsilon$ were $32.2 \%$ and $579.0 \%$ for Sample $\mathrm{C}$, in comparison to the $5.8 \%$ reduction in $\alpha$ and $118.0 \%$ increase in $\varepsilon$ for Sample D after the same 48-h duration of testing. The possible causes for such significant differences may include the differences in some key control parameters used in the manufacturing processes, such as the thickness of the coating, the pressure in the coating chamber, and the voltage used for the VMS [18]. Nevertheless, due to their excellent resistance to salt spray corrosion, Sample E and 
Sample F, which were manufactured with the same BCP technology but by different manufacturers, had essentially the same optical properties before and after the testing.

\section{Resistance to salt spray of SSACs by individual manufacturing technologies}

In this section, the resistance to salt spray of the SSACs by the three individual manufacturing technologies is further examined, using Samples A, C and E as the representative for each manufacturing technology, through the inspection of the physical appearance of the samples and the measured distribution of $\rho_{\lambda_{i}}$ over the solar spectrum before and after the salt spray testing over different durations of testing.

\section{Resistance to salt spray of SSACs by the AO technology}

The AO technology consists of putting aluminum strips or copper-aluminum composite strips into a dilute phosphoric acid solution to be anodized until a porous oxide film forms on the surfaces of the strips, followed by the alternating current electrolysis of the strips in a nickel sulfate or stannous sulfate solution, with the nickel or tin ions reduced and deposited on the oxidized pores in the film to form the desired SSAC.

Figure 6 presents the photos of Sample A before and after the salt spray testing over various durations. An inspection of the appearance of the coatings revealed that except for the slight deterioration of the luster after the salt spray testing, there were very little differences in the surfaces before and after the testing which can hardly be discerned by naked eyes. As discussed above and also shown in Table 1, the solar absorptance of Sample A did not change before and after the testing, although its thermal emittance increased by $15 \%$ after $12 \mathrm{~h}$ of testing. However, this thermal emittance value stayed the same when the duration of the salt spray testing was further prolonged.

The negligible impact of the salt spray testing on the solar absorptance of Sample A is clearly exhibited by the measured distributions of the monochromatic reflectance of solar radiation of Sample A over the solar spectrum before and after the salt spray testing over different durations, as shown in Fig. 7, which clearly demonstrates that the differences in $\rho_{\lambda_{i}}$ before and after the salt spray testing with various durations are negligible over the whole solar spectrum.

During the salt spray testing, the salt particles were sprayed into the pores of the coating where they reacted chemically with the substrate materials and air to form a thin film of $\mathrm{Al}_{2} \mathrm{O}_{3}$. The resultant corrosion led to the improvement of the smoothness of the coating surface which increased its thermal emittance, but deteriorated very slightly the luster of the coating surface. However, due to the protection of the $\mathrm{Al}_{2} \mathrm{O}_{3}$ film formed on the coating surface, further corrosion due to the chemical reaction between the salt particles and the substrate materials would be prevented, and hence any further prolonging of the salt spray testing duration would not change the thermal emittance of the sample.

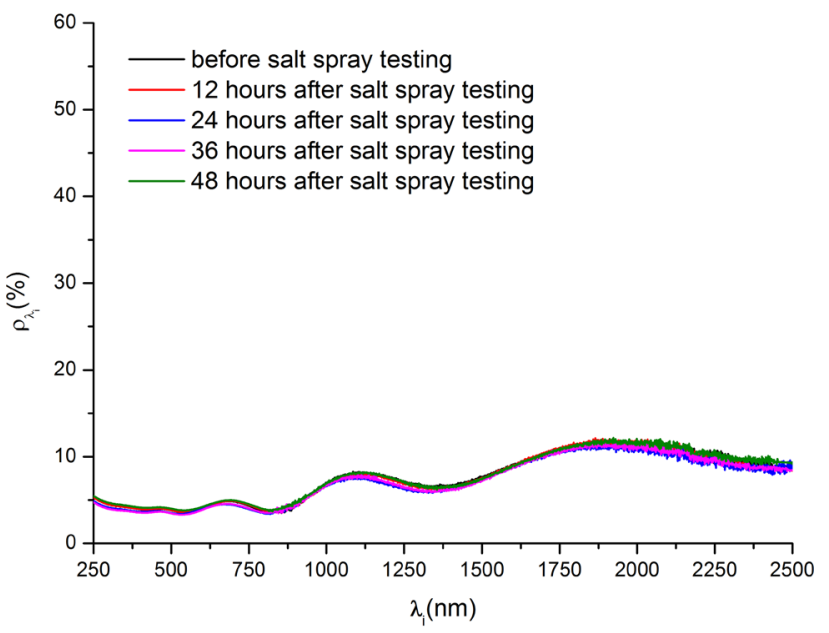

Fig. 7 The experimentally measured distributions of the monochromatic reflectance of solar radiation of Sample A over the solar spectrum before and after the salt spray testing over different durations

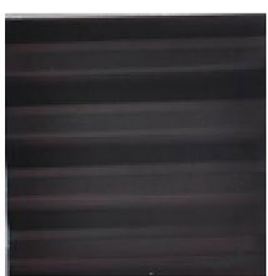

(a)

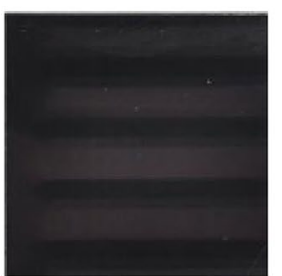

(b)

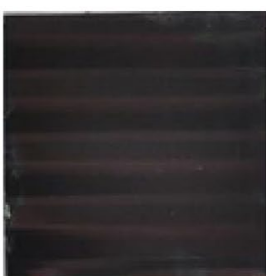

(c)

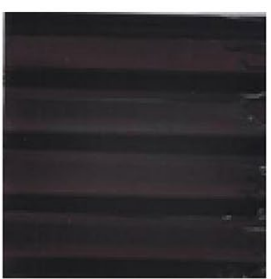

(d)

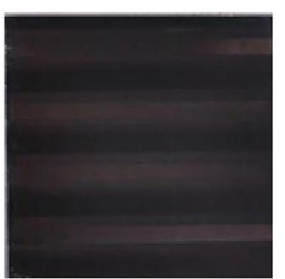

(e)

Fig. 6 The photos for Sample A before and after the salt spray testing over different durations: a before the testing, b after the 12-h duration, $\mathbf{c}$ after the $24-\mathrm{h}$ duration, $\mathbf{d}$ after the 36 -h duration, and e after the 48 -h duration 


\section{Resistance to salt spray of SSACs by the VMS technology}

The VMS technology consists of heating the coating material to its melting point in a vacuum chamber, and then using the ionized gas in the chamber to bombard the melted coating materials to be adhered to the substrate to form the SSAC [30].

The photos of Sample $\mathrm{C}$ before and after the salt spray testing over different durations are presented in Fig. 8. Significant changes of the physical appearance of the sample before and after the salt spray testing are very evident. Before the testing, the coating surface was smooth and free of cracks, blistering, peeling, or corrosion. After the testing, traces of corrosion of the coating surface were very obvious, and the severity of the corrosion and the sizes of the corrosion regions increased when the duration of the testing was prolonged. During the salt spray testing, initially the corrosion occurred only at some individual spots, and gradually these corrosion spots evolved into large lumps. After the 48-h salt spray testing, it was observed that the whole sample surface was corroded. As described above and shown in Table 1, both the solar absorptance and the thermal emittance of Sample C changed enormously, with $\alpha$ decreasing by $6.2,7.6,19.0$, and $28.9 \%$ and $\varepsilon$ increasing by 220,292 , 436 , and $579 \%$ after the testing durations of $12 \mathrm{~h}, 24 \mathrm{~h}, 36 \mathrm{~h}$, and $48 \mathrm{~h}$, respectively.

The significant impact of the salt spray testing on the solar absorptance of Sample C is obviously demonstrated by the measured distributions of $\rho_{\lambda_{i}}$ for Sample C over the solar spectrum before and after the salt spray testing over different durations, as shown in Fig. 9. It is seen that the values of $\rho_{\lambda_{i}}$ over the majority of the solar spectrum, in particular the whole spectrum region below $2000 \mathrm{~nm}$ where almost $99 \%$ of the solar energy resides, increased significantly after the salt spray testing and the amounts of increase in $\rho_{\lambda_{i}}$ increased markedly as well with the prolonging of the testing duration.

The significant adverse effects on the optical properties of the SSACs with the VMS technology are mainly caused by severe corrosion, which is the result of the chemical reaction between the salt particles and the coating surface materials

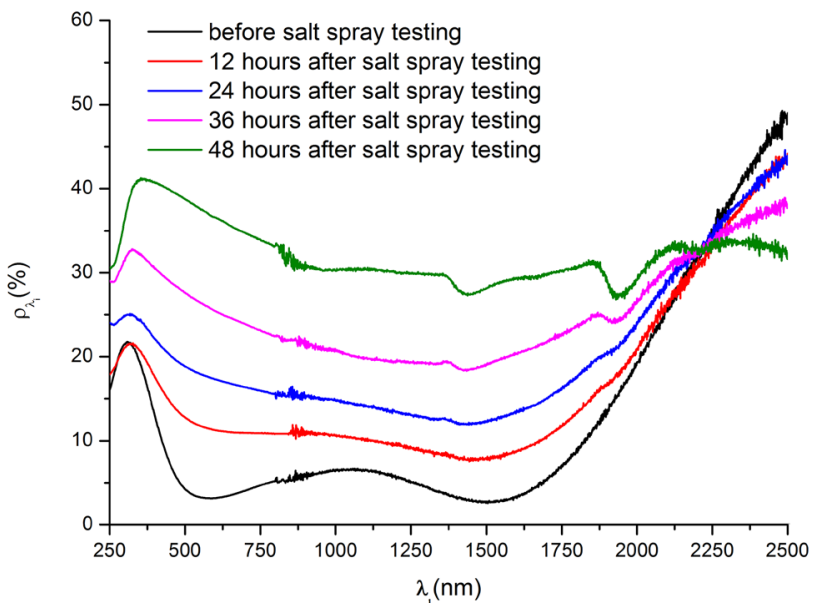

Fig. 9 The experimentally measured distributions of the monochromatic reflectance of solar radiation of Sample $\mathrm{C}$ over the solar spectrum before and after the salt spray testing over different durations

and the enhanced non-metallic properties of the substrate materials.

\section{Resistance to salt spray of SSACs by the BCP technology}

The BCP technology consists of electroplating black chromium on the metal substrate to form the selective black chromium layer. However, before the electroplating of black chromium on the metal substrate, a layer of copper or nickel is plated on the metal substrate first, which not only significantly increases the adhesion between the black chromium and the metal substrate, but also provides excellent resistance to corrosion and wear. Figure 10 presents the photos of Sample E before and after the salt spray testing over different durations. An inspection of the physical appearance of the coatings revealed a slight corrosion after the testing, which was limited to a few small spots, implying that the salt spray only has a minor effect on the SSACs with the BCP technology. As described above and shown in Table 1, the solar absorptance of Sample E was essentially unchanged after

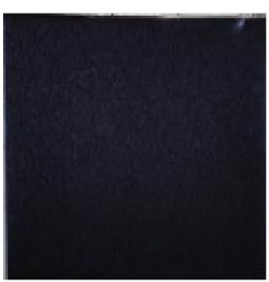

(a)

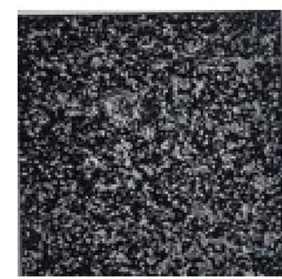

(b)

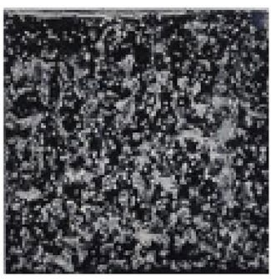

(c)

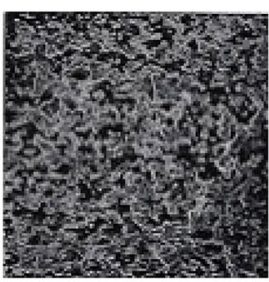

(d)

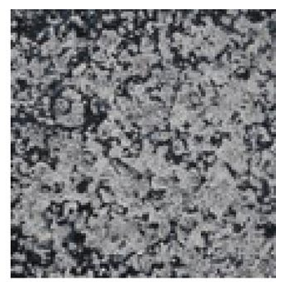

(e)

Fig. 8 The photos for Sample $\mathrm{C}$ before and after the salt spray testing over different durations: a before the testing, $\mathbf{b}$ after the 12-h duration, $\mathbf{c}$ after the 24 -h duration, $\mathbf{d}$ after the 36 -h duration, and $\mathbf{e}$ after the 48 -h duration 


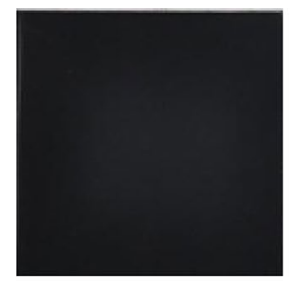

(a)

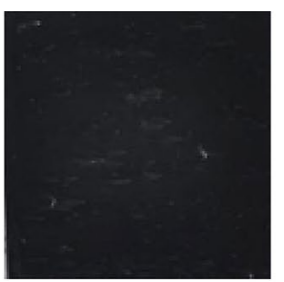

(b)

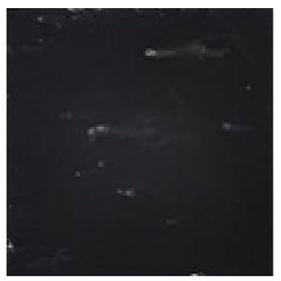

(c)

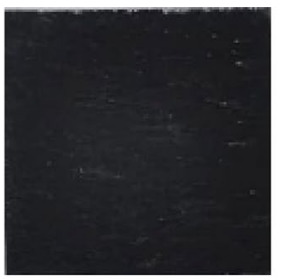

(d)

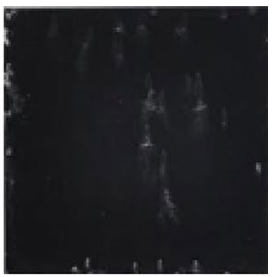

(e)

Fig. 10 The photos for Sample E before and after the salt spray testing over different durations: a before the testing, $\mathbf{b}$ after the 12-h duration, c after the 24-h duration, $\mathbf{d}$ after the 36 -h duration, and e after the 48 -h duration

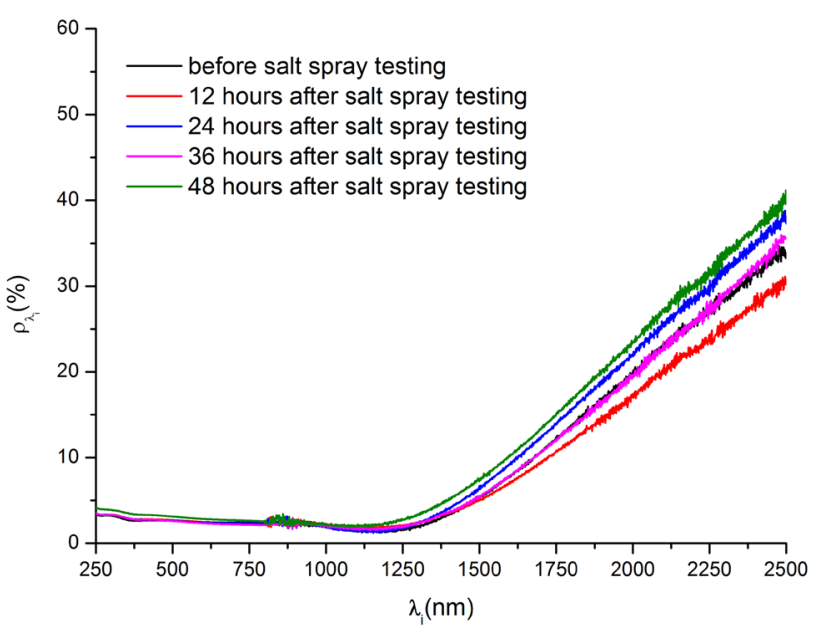

Fig. 11 The experimentally measured distributions of the monochromatic reflectance of solar radiation of Sample E over the solar spectrum before and after the salt spray testing over different durations

the salt spray testing, and its thermal emittance increased slightly after the 12-h testing but kept unchanged when the testing duration was further prolonged, indicating that the salt spray only has a minor effect on the optical properties of the SSACs with the BCP technology.

The minor impact of the salt spray testing on the solar absorptance of Sample E is also clearly exhibited by the measured distributions of $\rho_{\lambda_{i}}$ for Sample E over the solar spectrum before and after the salt spray testing over different durations, as shown in Fig. 11. It is seen that the differences in $\rho_{\lambda_{i}}$ before and after the salt spray testing with various durations over the region below $1200 \mathrm{~nm}$, in which the bulk of solar radiation resides, are negligible. Beyond $1200 \mathrm{~nm}$, the values of $\rho_{\lambda_{i}}$ differ before and after the testing and the differences increase with the wavelength. However, the contribution to $\alpha$ over this spectrum region is minimum, resulting in only very slight increases in $\varepsilon$ when the testing duration was prolonged.

\section{Conclusions}

The resistance to corrosion of six SSAC samples under an extended exposure to an adverse environment was evaluated by the salt spray testing, which is an accelerated aging test, aiming at examining the impact of salt spray on the optical properties of the SSACs manufactured by different manufacturing technologies, which include the AO technology, the VMS technology, and the BCP technology, over four testing durations of $12 \mathrm{~h}, 24 \mathrm{~h}, 36 \mathrm{~h}$, and $48 \mathrm{~h}$, respectively. The analysis of the impact of salt spray on the optical properties of the SSACs was also aided by the inspection of the photos of the selected SSAC samples and the experimentally measured distributions of the monochromatic reflectance of solar radiation of the samples over the solar spectrum before and after the salt spray testing over different durations.

The experimental results show that before the salt spray testing, all SSAC samples under consideration had good optical properties, but the samples manufactured with the BCP technology had the best overall optical properties, followed by those manufactured with the AO technology and then by those manufactured with the VMS technology. It was found that the salt spray has little effect on the solar absorptance of the SSACs manufactured with the AO and BCP technologies, but has a significant impact on the solar absorptance of those manufactured with the VMS technology. In terms of thermal emittance, although the salt spray was found to have only a minor effect on the SSACs manufactured with the BCP technology, it had a significant effect on those manufactured with the AO technology and an immerse effect on those manufactured with the VMS technology. Hence, in terms of the overall optical properties, when both the values of $\alpha$ and $\varepsilon$ are considered, it can be concluded that in general the SSACs manufactured with the $\mathrm{BCP}$ technology have excellent resistance to salt spray corrosion and those manufactured with the AO technology have only reasonable resistance to salt spray corrosion, whereas the SSACs manufactured with the VMS technology have very poor resistance to salt spray corrosion. The results also demonstrated that there are differences in the optical properties for the SSAC samples manufactured with the 
same technology but by different manufacturers, and some of these differences are very large. These differences were found to be caused by different causes.

Acknowledgements The support from the National Natural Science Foundation of China $(51866016,11662021)$ is gratefully acknowledged.

\section{Compliance with ethical standards}

Conflict of interest On behalf of all authors, the corresponding author states that there is no conflict of interest.

Open Access This article is distributed under the terms of the Creative Commons Attribution 4.0 International License (http://creativeco mmons.org/licenses/by/4.0/), which permits unrestricted use, distribution, and reproduction in any medium, provided you give appropriate credit to the original author(s) and the source, provide a link to the Creative Commons license, and indicate if changes were made.

\section{References}

1. Boubault, A., Ho, C.K., Hall, A., Lambert, T.N., Ambrosini, A.: Durability of solar absorber coatings and their cost-effectiveness. Sol. Energy Mater. Sol. Cells 166, 176-184 (2017)

2. Ma, F., Gao, W., Liu, T., Lin, W., Li, M.: An experimental study on the impacts of key parameters of all-glass evacuated tubes on the thermal performances of all-glass evacuated tube solar water heaters. J. Renew. Sustain. Energy 5, 023140 (2013)

3. Diamantino, T.C., Gonçalves, R., Nunes, A., Páscoa, S., Carvalho, M.J.: Durability of different selective solar absorber coatings in environments with different corrosivity. Sol. Energy Mater. Sol. Cells 166, 27-38 (2017)

4. Slamova, K., Duerr, I., Kaltenbach, T., Köhl, M.: Degradation effects of maritime atmosphere on metallic components of solar collectors. Sol. Energy Mater. Sol. Cells 147, 246-254 (2016)

5. Dan, A., Basu, B., Echániz, T., González de Arrietac, I., López, G.A., Barshilia, H.C.: Effects of environmental and operational variability on the spectrally selective properties of W/WAlN/ WAlON $/ \mathrm{Al}_{2} \mathrm{O}_{3}$-based solar absorber coating. Sol. Energy Mater. Sol. Cells 185, 342-350 (2018)

6. Diamantino, T.C., Carvalho, M.J., Nunes, A., Ferreira, C.: Durability of solar thermal collectors in environments with different corrosivity: inspection and fault analysis. Corros. Prot. Mater. 35(1), 15-20 (2016)

7. Koch, G.H., Brongers, M.P.H., Thompson, N.G., Virmani, Y.P., Payer, J.H.: Corrosion Costs and Preventive Strategies in the United States. [Online]. https://www.nace.org/uploadedfiles/ publications/ccsupp.pdf. Accessed 15 Dec 2015

8. International Organization for Standardization: ISO 9223:2012: Corrosion of Metals and Alloys-Corrosivity of AtmospheresClassification, Determination and Estimation, 2012. [Online]. https://www.iso.org/standard/53499.html. Accessed 15 Dec 2015

9. International Organization for Standardization: ISO 118443:2006: Corrosion of Metals and Alloys-Classification of Low Corrosivity of Indoor Atmospheres-Part 3: Measurement of Environmental Parameters Affecting Indoor Corrosivity, 2006. [Online]. https://www.iso.org/standard/36650.html. Accessed 15 Dec 2015
10. European Technology Platform on Renewable Heating and Cooling: Strategic Research Priorities for Solar Thermal Technology, 2012. [Online]. http://www.rhc-platform.org/fileadmin/ Publications/Solar_Thermal_SRP_single_page.pdf. Accessed 15 Dec 2015

11. Köhl, M., Carlsson, B., Jorgensen, S.E., Czanderna, A.: Performance and Durability Assessment: Optical Materials for Solar Thermal Systems, 1st edn. Elsevier, Oxford (2004)

12. Fernandes, J.C.S., Nunes, A., Carvalho, M.J., Diamantino, T.C.: Degradation of selective solar absorber surfaces in solar thermal collector-an EIS study. Sol. Energy Mater. Sol. Cells 160, 149-163 (2017)

13. International Energy Agency: Performance, Durability and Sustainability of Advanced Windows and Solar Components for Building Envelopes: Final Report, 2006. Project A2: Switchable Glazing, Subtask A: Performance, Task 27 Performance of solar façade components of Solar Heating and Cooling Programme. [Online]. https://www.iea-shc.org/data/sites/1/publications/ task27-a2.pdf. Accessed 10 Dec 2015

14. Roberge, P.R.: Corrosion Engineering: Principles and Practice. McGraw-Hill, New York (2008)

15. Carlsson, B., Möller, K., Köhl, M., Heck, M., Brunold, S., Frei, U., Marechal, J.-C., Jorgensen, G.: The applicability of accelerated life testing for assessment of service life of solar thermal components. Sol. Energy Mater. Sol. Cells 84(1-4), 255-274 (2004)

16. International Organization for Standardization: ISO/TR 16335:2013: Corrosion of Metals and Alloys-Corrosion Tests in Artificial Atmospheres-Guidelines for Selection of Accelerated Corrosion Test for Product Qualification, 2013. [Online]. https://www.iso.org/standard/56181.html. Accessed 12 Dec 2015

17. International Organization for Standardization: ISO 229753:2014: Solar Energy-Collector Components and Materials-Part 3: Absorber Surface Durability, 2014. [Online]. https ://www.iso.org/standard/61758.html. Accessed 12 Dec 2015

18. Carlsoon, B., Moller, K., Frei, U., Brunold, S., Köhl, M.: Comparison between predicted and actually observed in-service degradation of a nickel pigmented anodized aluminium absorber coating for solar DHW systems. Sol. Energy Mater. Sol. Cells 61(3), 223-238 (2000)

19. Köhl, M.: Durability of solar energy materials. Renew. Energy 24, 597-607 (2001)

20. Zhang, K., Hao, L., Du, M., Mi, J., Wang, J., Meng, J.: A review on thermal stability and high temperature induced ageing mechanisms of solar absorber coatings. Renew. Sustain. Energy Rev. 67(C), 1282-1299 (2017)

21. Leygraf, C., Wallinder, I.O., Tidblad, J., Graedel, T.: Atmospheric Corrosion, 2nd edn. Wiley, Oxford (2000)

22. The Chinese National Standards Bureau: GB/T 26974-2011: Specification for the Absorbers of Flat-Plate Solar Collectors, 2011. [Online]. http://www.gbstandards.org/China_standards/ GB/GB_T\%2026974-2011.htm. Accessed 10 Aug 2014

23. Fan, J., Zhou, X., Zhou, S., Qin, X.: A brief introduction to the technological issues for flat-plate solar collectors. Chin. Sol. Energy 2013(5), 30-32 (2013)

24. Yao, K., Song, K., Wang, G., Liang, M., Xia, Z., Fan, J.: A discussion on the resistance performance to salt spray of solar selective absorber coatings. Chin. Sol. Energy 2013(7), 20-22 (2013)

25. Yin, Z., Zhou, X., Wei, B.: Evaluating the resistance performance to salt spray of solar selective absorber coatings. Chin. Sol. Energy 2013(9), 12-15 (2013)

26. The Chinese National Standards Bureau: GB/T 1771-2007: Paints and Varnishes-Determination of Resistance to Neutral Salt Spray (fog), 2007. [Online]. http://www.gbstandards.org/ 
China_standards/GB/GB_T\%201771-2007.htm. Accessed 5 Aug 2014

27. Duffie, J.A., Beckman, W.A.: Solar Engineering of Thermal Processes, 2nd edn. Wiley, Oxford (1991)

28. The Chinese National Standards Bureau: GB/T 25965-2010: Test Methods of Normal Emittance of Materials and Hemispherical of All-Glass Evacuated Collector Tubes, 2010. [Online]. http://www.gbstandards.org/China_standards/GB/ GB_T\%2025965-2010.htm. Accessed 21 Jul 2014

29. The International Organization for Standardization: ISO 3696:1987: Water for Analytical Laboratory Use-Specification and Test Methods, 1987. [Online]. https://www.iso.org/stand ard/9169.html. Accessed 16 Aug 2014

30. Kong, L., Fan, D., Wang, C., Ling, X., Lanzhou, J.: Processing technology of spectrally selective absorption coatings used for flat collector. J. Lanzhou Jiaotong Univ. 32(3), 62-65 (2013)

Publisher's Note Springer Nature remains neutral with regard to jurisdictional claims in published maps and institutional affiliations. 